\title{
Review of: "Li-ion storage properties of two- dimensional titanium-carbide synthesized via fast one-pot method in air atmosphere"
}

$\mathrm{Lu} \mathrm{Li}{ }^{1}$

1 Harbin Normal University

Potential competing interests: The author(s) declared that no potential competing interests exist.

Since the first report of Ti3C2Tx synthesis in 2011, MXenes have typically been prepared from selectively etching MAX phase precursors. However, these methods are difficult to upscale and/or take hours to days to obtain MXenes, which greatly reduces production efficiency and increases production costs. In this paper $\square$ the author proposes a facile one-pot synthesis method for preparing MXenes from elemental precursors in an air atmosphere. By eliminating the need for inert gas protection, the synthesis operation is greatly simplified, and the one-pot method reduces the whole synthesis duration compared to conventional MXene synthesis methods that prepare MAX phase precursors and MXenes separately. Cl-terminated Ti3C2Tx and T2CTx MXenes are prepared with a fast-etching step at $700{ }^{\circ} \mathrm{C}$ for only 10 mins and a whole synthesis duration of less than $8 \mathrm{~h}$. The one-pot synthesis method paves the way for the facile and fast synthesis of MXene materials with lower production costs and sheds light on the promising potential of MXene materials for energy storage applications. 